\title{
ESTUDO DO COMPORTAMENTO DINÂMICO DO MODELO NEURONAL DE HINDMARSH-ROSE
}

\section{STUDY OF THE DYNAMIC BEHAVIOR OF THE HINDMARSH-ROSE NEURONAL MODEL}

Raildo Santos de Lima; Fábio Roberto Chavarette ${ }^{2}$; Luiz Gustavo Pereira Roéfero $^{3}$

\footnotetext{
${ }^{1}$ Universidade Federal de Mato Grosso do Sul - UFMS, Departamento de Matemática, Paranaíba-MS. ${ }^{2}$ Universidade Estadual Paulista - UNESP, Faculdade de Engenharia de Ilha Solteira, Departamento de Matemática. ${ }^{3}$ Discente da Universidade Estadual Paulista - UNESP, Faculdade de Engenharia de Ilha Solteira, Departamento de Engenharia Mecânica

E-mail: Igpr98gu@gmail.com, fabio.chavarette@unesp.br, raildo.lima@ufms.br
}

RESUMO - Baseado no modelo neuronal de Hindmarsh-Rose (HR) para transmissão de impulsos nervosos, este trabalho visa estudar propriedades e o comportamento dinâmico do sistema caótico não linear que descreve o bursting neuronal em um único neurônio. Por parte da bioengenharia, existe grande motivação no estudo do modelo HR pelo fato de ser bem representativo ao neurônio biológico, podendo, assim, simular vários comportamentos de um neurônio real, dentre eles, o comportamento periódico, aperiódico e caótico. A literatura sugere que o comportamento caótico represente no ser humano o estado epilético ou convulsivo. Através de simulações computacionais, considerando os parâmetros do sistema, foi analisado que a estabilidade é altamente sensível as condições iniciais e produzindo oscilações, mais ainda, quando a oscilação aumenta o comportamento aleatório tende a aumentar tornando o sistema imprevisível.

Palavras-chave: Modelo de Hindmarsh-Rose; Dinâmica Neuronal; Estabilidade.

ABSTRACT - Based on the Hindmarsh-Rose (RH) neuronal model for nerve impulse transmission, this paper aims to study the properties and dynamic behavior of the non-linear chaotic system that describes neuronal bursting in a single neuron. On the part of bioengineering, there is great motivation in the study of the HR model because it is well representative of the biological neuron, being able to simulate several behaviors of a real neuron, among them periodic, aperiodic and chaotic behavior. The literature suggests that the chaotic behavior represents in the human being the epileptic or convulsive state. Through computer simulations, considering the system parameters, it was analyzed that the stability is highly sensitive to the initial conditions and producing 
oscillations, more so, when the oscillation increases the random behavior tends to increase making the system unpredictable.

Keywords: Hindmarsh-Rose model; Neuronal Dynamics; Stability. 


\section{INTRODUÇÃO}

O sistema nervoso controla todas as atividades físicas conscientes e inconscientes. Ele é formado por bilhões de células nervosas que captam informações vindas do interior e exterior do corpo humano. As células nervosas, bem como as musculares, têm duas propriedades fisiológicas características que são a excitabilidade e condutibilidade, ou seja, capacidade de reagir a um dado estímulo e transmitir esse estímulo sob a forma de impulsos eletroquímicos ao longo de suas membranas. A membrana das células nervosas possui propriedades elétricas que são dadas através do arranjo molecular de seus componentes. Estas propriedades podem afetar a capacidade das células eletricamente excitáveis na condução das informações e determinam a sequência de alterações de voltagem produzidas pelo fluxo de corrente através das membranas. A membrana do neurônio é frequentemente comparada a um circuito elétrico que compreende muitas unidades repetidas. Cada unidade contém certo número de baterias, um para cada espécie de íon, sendo as forças eletromotrizes dessas baterias determinadas pelos gradientes de concentração para os diferentes íons. No análogo elétrico, cada bateria iônica está ligada às fases extra e intracelular por uma resistência variável, que representa a permeabilidade da membrana relativa à espécie de íon em questão. Para estudar o comportamento da membrana durante o repouso e a passagem de impulsos nervosos modelos matemáticos foram construídos, entre eles o modelo de Hodgkin e Huxley publicado em 1952, apresentando os resultados de uma sequência de experimentos nos quais investigaram o fluxo de corrente elétrica através da superfície da membrana da fibra nervosa de uma lulagigante. Os autores desenvolveram uma descrição matemática para o comportamento da membrana e baseada nestas experiências as explicam a condução e a excitação da fibra. As fórmulas desta descrição têm sido usadas desde então como a base para quase todos os outros modelos de tecidos excitáveis que envolvem correntes iônicas (HODGKIN; HUXLEY, 1952).

O objetivo deste trabalho é simular o comportamento caótico através do método numérico Runge Kutta de $4^{\mathrm{a}}$ ordem (RUGGIERO; LOPES, 1996) aplicado ao modelo neuronal de Hindmarsh-Rose (HR) (HINDMARSH; ROSE, 1984).

Este trabalho está organizado como a seguir. Na seção 2 apresenta-se o modelo matemático proposto em (HINDMARSH; ROSE, 1984). As simulações numéricas do modelo proposto com comportamento caótico estão na seção 3 . Na seção 4 apresentam-se as considerações finais com agradecimentos e logo em seguida as referências utilizadas.

\section{MODELO MATEMÁTICO}

Basicamente, um modelo de sistema fisiológico é uma representação da realidade, porém, existe um grau de aproximação. Modelos podem ter formas, ser conceituais, estatísticos, dentre outros. O modelo é um objeto importante na formulação da metodologia do processo de modelagem, pois através do modelo pode-se descrever, interpretar, predizer e explicar o sistema. (MONTEIRO, 2002).

A literatura regista que o primeiro modelo matemático relativamente completo da dinâmica envolvendo a membrana neurônica, foi publicado por Hodgkin e Huxley em 1952. Na década de 50, FitzHugh reduziu o modelo de Hodgkin-Huxley para um modelo de duas variáveis para realizar uma análise do plano de fase. Este modelo fornece um espaço de fase de explicação qualitativa de formação e decadência do potencial de ação, tal modelo é uma simplificação bem conhecida na literatura na qual foi sugerida por FitzHugh (FITZHUGH, 1961) e usada por Nagumo et al. (NAGUMO et al., 1962) denominada modelo de Fitzhugh-Nagumo. 
Hindmarsh e Rose, inicialmente em 1982, descreveram uma modificação do modelo de Hodgkin-Huxley na qual introduziram dois pontos de equilíbrio adicionais e posteriormente, em 1984, descreveram uma modificação do próprio modelo desenvolvido por eles em que, desta vez, mostraram que um destes pontos de equilíbrio é um ponto de sela gerando assim, duas separatrizes que divide o plano de fase em duas regiões. Em uma região, todas as órbitas se aproximam de um ciclo limite e na outra região, todas as órbitas se aproximam de um ponto de equilíbrio estável. (HINDMARSH; ROSE, 1984).

Quando algum estímulo seja químico, mecânico ou elétrico chega ao neurônio, pode ocorrer uma alteração da permeabilidade da membrana permitindo na célula nervosa grande entrada de sódio e pequena saída de potássio. Isso ocorre com uma inversão das cargas ao redor da membrana tornando-a despolarizada, fato que leva a um potencial de ação. Essa diferença de potencial varia de acordo com a quantidade de íons no meio intracelular e tem um valor estacionário de $-70 \mathrm{mV}$. O impulso nervoso se caracteriza pela propagação dessa despolarização pelo neurônio, com isso, logo após a passagem do impulso a membrana sofre uma repolarização recuperando seu estado normal de repouso cessando a transmissão do impulso.

Na Figura 1 estão ilustradas algumas partes principais do neurônio. Os Dendritos são as ramificações na qual o neurônio recebe estímulos provenientes de outras células. O Axônio é o prolongamento do corpo celular por onde se propaga um impulso nervoso para que este impulso interaja com outros neurônios ou fibras musculares. A Bainha de Mielina tem como função proteger o Axônio e também acelerar a velocidade da condução do impulso nervoso.

Figura 1. Modelo Biológico.

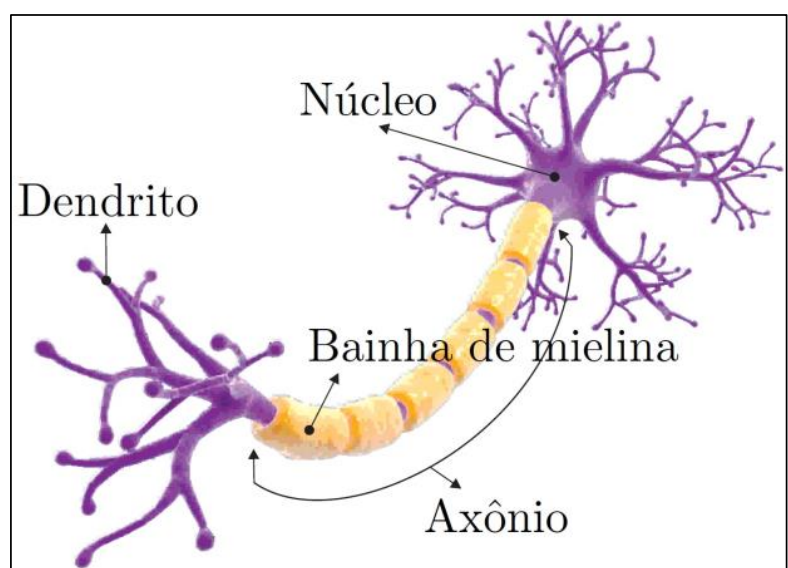

Fonte: https://www.turbosquid.com/pt br/3d-models/neuron-3d-max/819986

A geração de burstings neuronais tem sido extensivamente estudada no contexto do modelo neuronal de Hindmarsh-Rose $(H R)$, o qual estabelece uma variável de estado adimensional para o potencial de membrana $x(\mathrm{t})$ e outras duas (também adimensionais), $y(\mathrm{t})$ associada aos fluxos iônicos rápidos gerados pelo transporte de $\mathrm{Na}^{+}$e $\mathrm{K}^{+}$e $z(\mathrm{t})$ que captura a dinâmica de outros canais lentos. Essas variáveis se relacionam de forma não linear na composição do potencial de membrana, o que pode ser traduzido pela seguinte dinâmica (HINDMARSH,; ROSE, 1984):

$$
\left\{\begin{array}{l}
\dot{x}=y-a x^{3}+b x^{2}-z+I ; \\
\dot{y}=c-d x^{2}-y ; \\
\dot{z}=r\left[s\left(x-x_{r}\right)-z\right],
\end{array}\right.
$$


onde $a, b, c, d, s, r, x_{r}$ e $I$ são parâmetros do sistema que dependendo dos valores adotados, permitem simular um vasto conjunto de comportamentos dinâmicos topologicamente equivalentes aos observados no âmbito experimental. Isto faz do modelo HR um dos mais emblemáticos no estudo qualitativo do fenômeno de bursting neuronal (BUTERA et al., 1999). O sistema apresenta vários comportamentos dentre eles, um típico comportamento caótico do potencial de membrana, isto é, um comportamento aperiódico e com sensibilidade em relação às condições iniciais adotando-se a estimulação externa constante I como um parâmetro de controle.

\section{SIMULAÇÕES NUMÉRICAS}

Os parâmetros adimensionais adotados para as simulações numéricas são $a=1, b=3, c=1, d=5, r=0,006, s=4$, $x_{r}=-1,56$ e variando o parâmetro $I$ em cada análise de simulação. Estes parâmetros são definidos de maneira conveniente para um melhor entendimento do bursting neuronal (HINDMARSH; ROSE, 1984).

Figura 2. Comportamento Dinâmico com Parâmetro $I=1,1$.

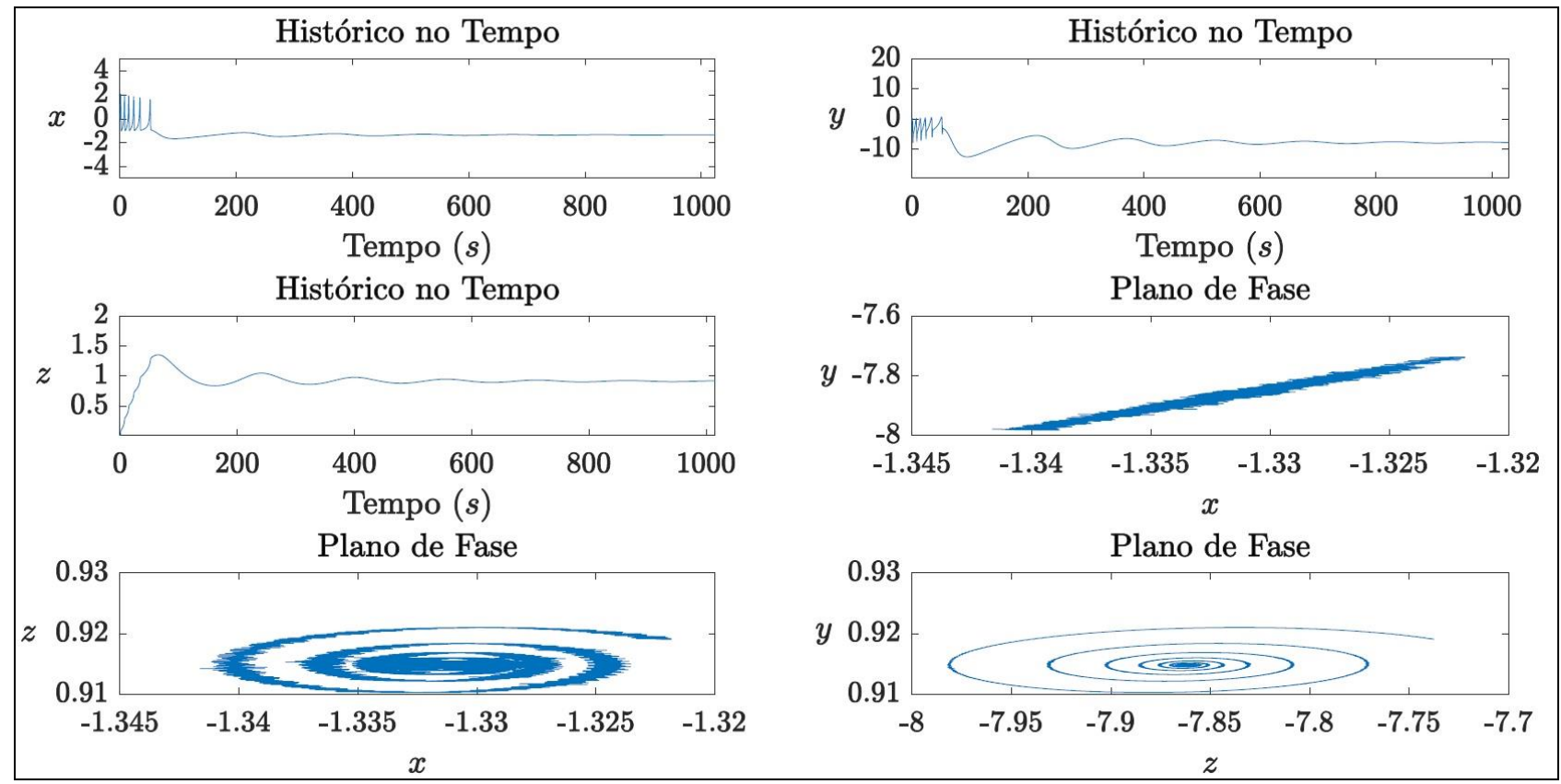

Fonte: Os autores.

O comportamento dinâmico (MONTEIRO, 2002; SAVI, 2006), ilustrado na Figura 2, representa a fase de silêncio do neurônio onde o comportamento do sistema converge para um ponto estável. Mais especificamente, tal órbita periódica altera entre duas fases bem definidas que são a fase ativa representando o momento inicial transiente e a fase de silêncio representando as oscilações com amplitude quase nula. Tais fases correspondem aos valores alto e baixo da variável $x$.
A Figura 3 ilustra o comportamento adquirido pelo neurônio após ser excitado pelo parâmetro constante $I$, tornando a resposta do neurônio em fase de pulsos, ou seja, com comportamento oscilatório.

A Figura 4 ilustra um comportamento aleatório, instável e caótico que no sistema representa a fase de pulsos aleatórios, sendo biologicamente comparada a um indivíduo com epilepsia. Como os neurônios se comunicam por impulsos elétricos ou por neurotransmissores, nas pessoas com epilepsia essa comunicação elétrica é 
exagerada liberando muitos neurotransmissores e com isso, os neurônios disparam de forma aleatória gerando a crise epilética
(CHÁVEZ et al., 2003; DA SILVA et al., 2003; MILAN, 2001).

Figura 3. Comportamento Dinâmico com Parâmetro $I=1,2$.

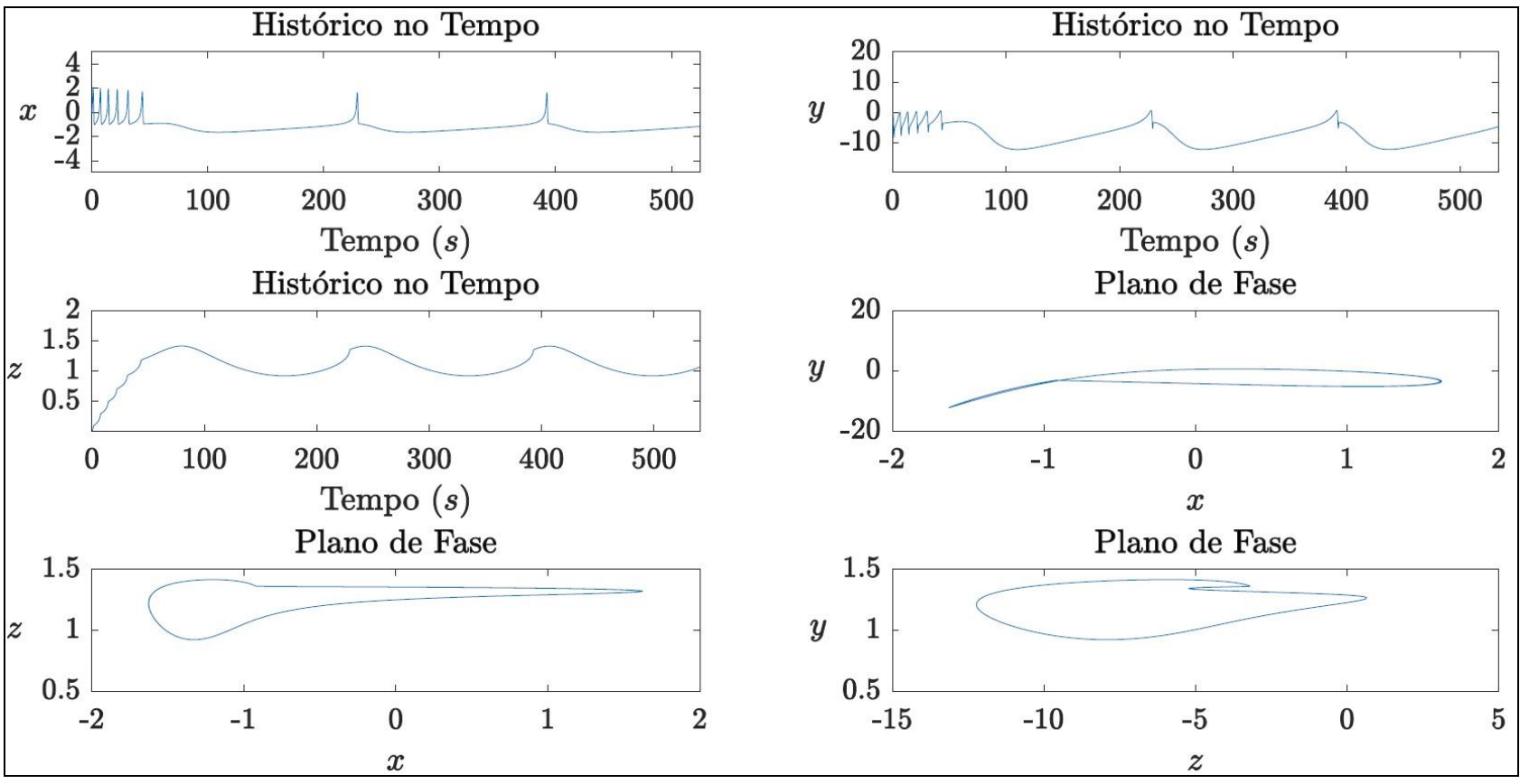

Fonte: Os autores.

Os expoentes de Lyapunov são usados como indicadores de caos em um determinado sistema. Estes expoentes indicam se uma órbita é sensível ou não às condições iniciais. Se duas órbitas com condições iniciais próximas divergem exponencialmente ao longo das iteradas, então as órbitas são classificadas como caóticas.

Como ilustrado na Figura 5, foi analisado os expoentes de Lyapunov para o sistema (1) e para tais condições foram encontrados os seguintes expoentes de
Lyapunov: $\quad \lambda_{1}=0,012, \quad \lambda_{2}=0,004 \quad \mathrm{e}$ $\lambda_{3}=-0,596$, na qual de acordo com (WOLF et al, 1985) a existência de pelo menos um expoente de Lyapunov positivo caracteriza o comportamento caótico do sistema.

A excitação externa $I$, neste trabalho, é utilizada como parâmetro de controle do sistema, sendo possível a demonstração dos diversos comportamentos que descrevem o sistema. 
Figura 4. Comportamento Dinâmico com Parâmetro $I=1,3$.

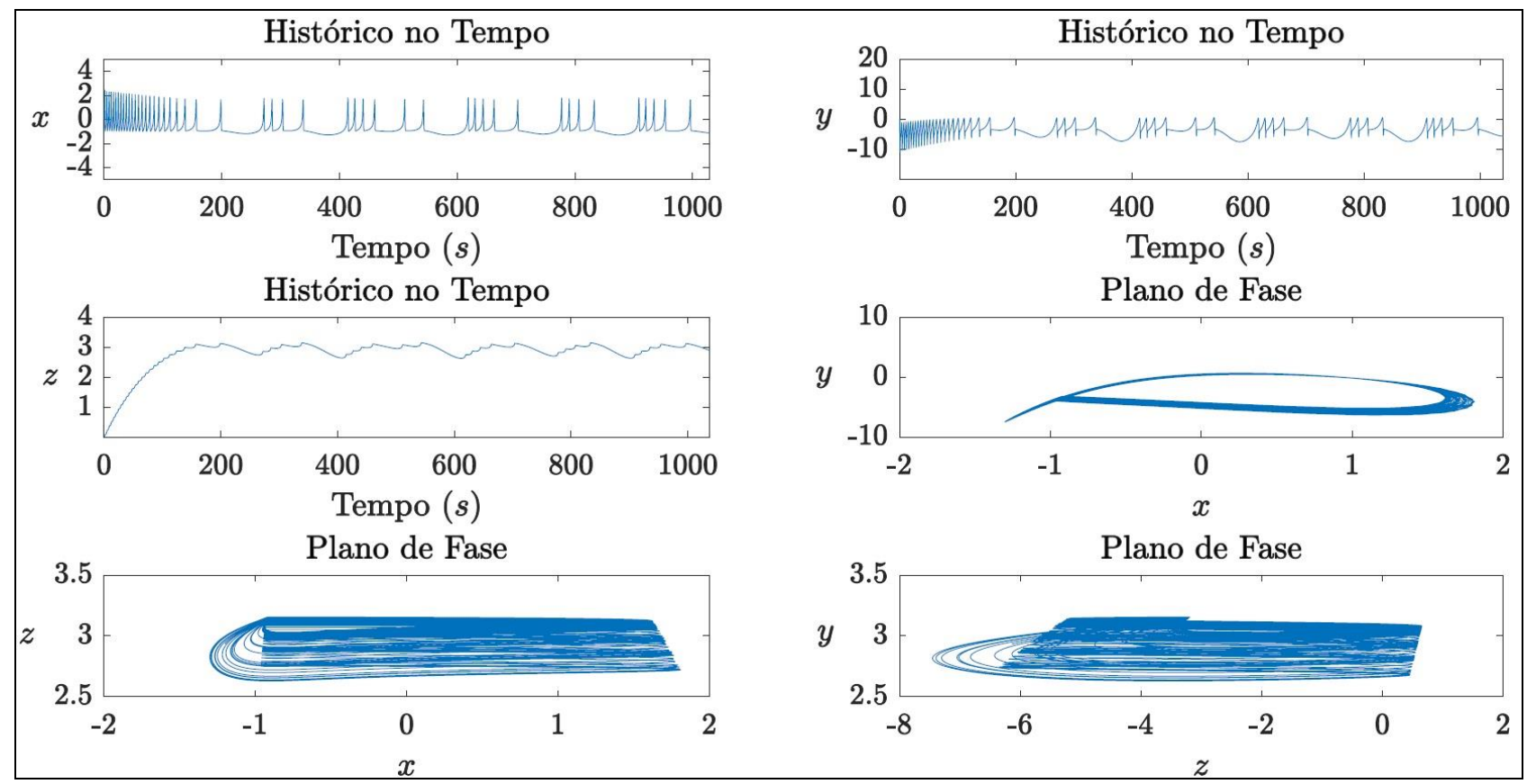

Fonte: Os autores.

Figura 5. Existência de Expoente de Lyapunov Positivo

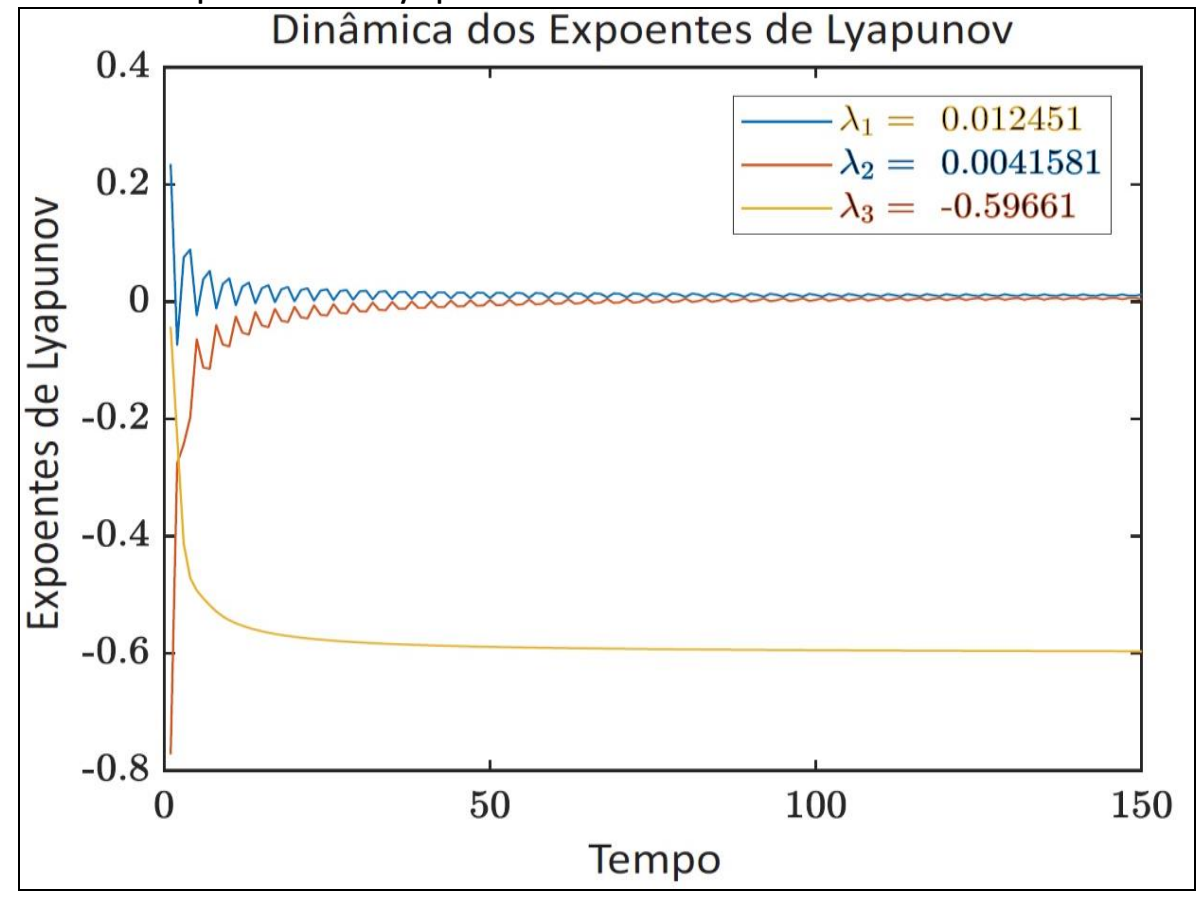

Fonte: Os autores.

\section{CONSIDERAÇÕES FINAIS}

Com o auxílio de simulações computacionais obteve-se vários comportamentos sobre o sistema estudado. Verifica-se, para determinados valores da corrente $I$, que o sistema apresenta comportamento periódico porém, aumentando cada vez mais o parâmetro constante $I$ o sistema evolui de maneira a perder sua periodicidade adquirindo aleatoriedade e imprevisibilidade (caos), concluindo a sensibilidade do sistema às condições iniciais. Esta sensibilidade às condições iniciais produzindo potenciais de ação com tempo de duração não determinado são observados em neurônios 
biológicos, fato que desperta grande interesse por parte da bioengenharia.

$\mathrm{Na}$ intenção de proporcionar maior clareza, todas as três projeções dos planos de fase foram apresentadas para melhor análise dos diversos comportamentos do sistema.

Aproximadamente 50 milhões de pessoas em todo o mundo sofrem de epilepsia. Os números divulgados pela Organização Mundial da Saúde (OMS), posicionam a epilepsia como uma das doenças neurológicas mais comuns no planeta (dados de 2017).

Neste ensejo, pesquisas futuras poderão proporcionar descobertas e métodos mais eficientes no tratamento da epilepsia, como por exemplo, medicamentos com melhor tempo de resposta e até mesmo a criação de dispositivos controladores do bursting neuronal.

\section{AGRADECIMENTOS}

Agradeço ao laboratório SISPLEXOS por ceder o espaço físico e o parque computacional para as simulações realizadas neste trabalho.

\section{REFERÊNCIAS}

CHÁVEZ, M.; QUYEN, L. V.; NAVARRO, M.; BAULAC, V.; MARTINERIE, J. Spatio-temporal dynamics prior to neocortical seizures: amplitude versus phase couplings. IEEE Transactions on Biomedical Engineering, v. 50, n. 5, p. 571-583, 2003. https://doi.org/10.1109/TBME.2003.810696

da SILVA, F. H. L. et al. Dynamical diseases of brain systems: different routes to epileptic seizures. IEEE transactions on biomedical engineering, v. 50, n.5, p. 540-548, 2003. https://doi.org/10.1109/TBME.2003.810703

FITZHUGH, R. Impulses and physiological states in theoretical models of nerve membrane. Biophysical journal, v. 1, n. 6, p. 445-466, 1961. https://doi.org/10.1016/S00063495(61)86902-6
HINDMARSH, J. L.; ROSE, R. A model of neuronal bursting using three coupled first order differential equations. Proc. R. Soc. Lond. B, The Royal Society, v. 221, n. 1222, p. 87-102, 1984. https://doi.org/10.1098/rspb.1984.0024

HODGKIN, A. L.; HUXLEY, A. F. A quantitative description of membrane current and its application to conduction and excitation in nerve. The Journal of physiology, v. 117, n. 4, p. 500-544, 1952. https://doi.org/10.1113/iphysiol.1952.sp004 $\underline{764}$

BUTERA JR, R. J.; RINZEL, J.; SMITH, J. C. Models of respiratory rhythm generation in thepre-botzinger complex. I. Bursting pacemaker neurons. Journal of neurophysiology, American Physiological Society Bethesda, v. 82 , n. 1, p. 382-397, 1999.

https://doi.org/10.1152/in.1999.82.1.382

MILAN, P. et al. Synchronization and information flow in EEGs of epileptic patients. IEEE Engineering in Medicine and Biology Magazine, v. 20, n. 5, p. 65-71, 2001. https://doi.org/10.1109/51.956821

MONTEIRO, L. H. A. Sistemas Dinâmicos. São Paulo: Livraria da Física, 2002.

NAGUMO, J.; ARIMOTO, S.; YOSHIZAWA, S. An active pulse transmission line simulating nerve axon. Proceedings of the IRE, v. 50, $n$. 10, p. 2061-2070, 1962. https://doi.org/10.1109/JRPROC.1962.28823 $\underline{5}$

RUGGIERO, M. A. R.; LOPES, V. L. R. Cálculo Numérico: Aspectos teóricos e computacionais. 2. ed. São Paulo:Pearson, 1996.

SAVI, M. A. Dinâmica não-linear e caos, São Paulo: Ed. E-papers, 2006. 
WOLF, A.; SWIFT, J. B.; SWINNEY, H. L.;

VASTANO, J. A. Determining Lyapunov

Exponents from a Time Series, Physica, v.

16D, p. 285-317, 1985.

https://doi.org/10.1016/0167-

2789(85)90011-9 